\title{
Image of the month: Gastropericardial fistula
}

\author{
Authors: Matteo Gandalini, ${ }^{A}$ Sylke Liebig, ${ }^{B}$ Felix Zwicker, ${ }^{C}$ Marcus Schuchmann ${ }^{D}$ and Markus Juchems ${ }^{E}$
}
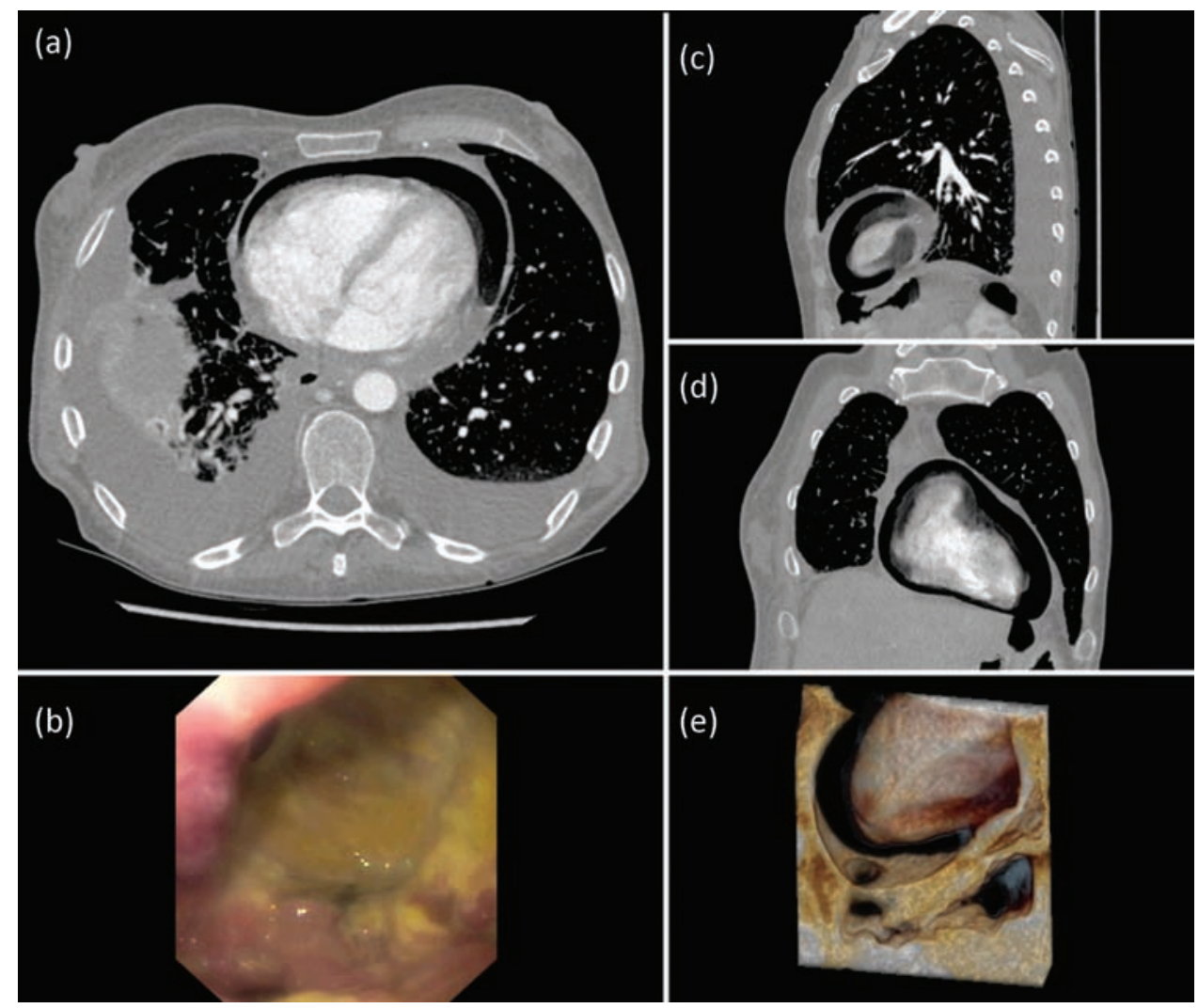

Fig 1. Computed tomography (CT) scan of chest and abdomen: axial (a), sagittal (c) and coronal (d) views demonstrating air in pericardial sac due to a fistula between the stomach and the pericardium. The $\mathrm{CT}$ scan shows in addition bilateral pleural effusions and right lung dystelectases. Endoscopic image of the fistula, view from the gastric antrum (b). Three dimensional CT reconstruction with depiction of the communication between the stomach and the pericardium (e).

A 50-year-old woman affected by an ovarian cancer (Figo IV) was referred to our Radiotherapy Department. She had a history of a slow disease progression for over 6 years as a result of several adjuvant chemotherapy regimens. During the last

Authors: A radiology resident, Department of Radiotherapy, Klinikum Konstanz and Radiology Practice, Konstanz, Germany; ${ }^{B}$ consultant in radiotherapy, Department of Radiotherapy, Klinikum Konstanz and Radiology Practice, Konstanz, Germany;

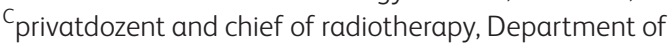
Radiotherapy, Klinikum Konstanz and Radiology Practice, Konstanz,

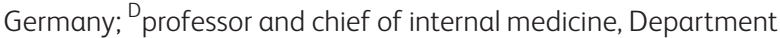
of Medicine, Klinikum Konstanz, Konstanz, Germany; ${ }_{\text {Eprofessor and }}$ chief of radiology, Department of Diagnostic and Interventional Radiology, Klinikum Konstanz, Konstanz, Germany chemotherapy session, she developed a gastric outlet obstruction caused by peritoneal metastases around the antrum and, as a result, she underwent a laparotomy with a Roux-en-Y side-to-side gastroenterostomy of the gastric fundus. After 2 months, multiple cerebral metastases were diagnosed and in order to treat them palliative whole brain radiotherapy was started. During this phase, the patient developed increasing abdominal and chest pain over a period of 3 days. Physical examination revealed sparse bowel sounds and constipation as well as a progressive decline in blood pressure. A blood sample showed an increase in C-reactive protein and a slight reduction in kidney function. A computed tomography scan (CT) of the chest and abdomen detected a transperitoneal communication between the stomach and the pericardium as well as a pathological air collection in the pericardial sac. The patient was transferred to the intensive care unit where a gastroscopy confirmed the diagnosis of gastropericardial fistula (Fig 1). Even though the possibility of a surgical closure of the fistula was 
discussed with the patient, she refused all treatment owing to the poor prognosis associated with such an advanced cancer. All therapies were consequently halted and the patient was discharged to home, where she died a week later.

Gastropericardial fistula is a rare condition associated with a high mortality rate and characterised by a direct communication between the stomach and the pericardium. Its etiology includes previous gastrointestinal surgery, perforating gastric ulcer and perforating cancer. ${ }^{1-2}$ In this case the perforation was due to an ulcerated peritoneal metastasis around the antrum and duodenal bulb. Although it is uncommon, gastropericardial fistula should be considered alongside pulmonary embolism and ileus in patients affected by peritoneal metastasis whenever they present with unexplained insidious chest and abdominal pain with hypotension. In these patients, a CT of the chest and abdomen can be helpful in providing the diagnosis.

\section{Consent to publish}

Consent to publish was obtained from the patient's next of kin.

\section{References}

1 Azzu V. Gastropericardial fistula: getting to the heart of the matter. BMC Gastroenterol 2016:16:96.

2 Imran Hamid U, Booth K, McManus K. Is the way to a man's heart through his stomach? Enteropericardial fistula: case series and literature review. Dis Esophagus 2013;26:457-64.

Address for correspondence: Mr Matteo Gandalini, Radiotherapy and Radiology Practice, Haydnstraße 2, Konstanz 78464, Germany.

Email: gandalini@radiologen-konstanz.de

\title{
Quacks, rogues and charlatans
}

\author{
Quacks, rogues and charlatans takes the reader on a \\ lively tour of colourful characters and dubious cures \\ that have littered the Royal College of Physicians' \\ 500-year history.
}

Order by phone or online

$\mathrm{Tel}+44(0) 2030751358$

or visit www.rcplondon.ac.uk/shop
Price: $€ 12$

(Postage and packaging not included)

$10 \%$ discount for fellows and members.

$15 \%$ discount for foundation doctors and students 